\title{
Methods in Pediatric Sleep Research and Sleep Medicine
}

\author{
Anne-Laure Mouthon ${ }^{1,2}$ Reto Huber ${ }^{1,3}$ \\ ${ }^{1}$ Child Development Center and Pediatric Sleep Disorders Center, \\ University Children's Hospital, Zurich, Switzerland \\ 2 Pediatric Rehab Research Group, Rehabilitation Center Affoltern am \\ Albis, University Children's Hospital, Zurich, Switzerland \\ ${ }^{3}$ University Clinics for Child and Adolescent Psychiatry, University of \\ Zurich, Zurich, Switzerland
}

Neuropediatrics 2015;46:159-170.

\begin{abstract}
Address for correspondence Reto Huber, PhD, Child Development Center and Pediatric Sleep Disorders Center, University Children's Hospital, Steinwiesstrasse 75, CH-8032 Zurich, Switzerland (e-mail: reto.huber@kispi.uzh.ch).
\end{abstract}

\author{
Abstract \\ Keywords \\ - sleep \\ - development \\ - questionnaires \\ - actigraphy \\ - polysomnography \\ - electroence- \\ phalography
}

\begin{abstract}
Several methods are used to evaluate sleep in infants, children, and adolescents including: Questionnaires and diaries, actigraphy, polysomnography, and electroencephalography which are well established. Novel approaches such as high-density electroencephalography, simultaneous electroencephalography-functional magnetic resonance imaging and nonpharmacological methods aiming for a modulation of sleep are currently only used for research. These approaches might become valuable methods for clinical application in the future. The purpose of this review is to present an overview of current methods and their respective fields of application and to report available rules and recommendations for their use.
\end{abstract}

\section{Introduction}

In pediatric sleep medicine clinicians assess sleep to identify sleep problems and to diagnose sleep disorders. Sleep problems such as bedtime problems, night wakings, and poor sleep hygiene are highly prevalent in the pediatric population. It has been reported that approximately $25 \%$ of all children experience some type of sleep problem, at least once during childhood however, sleep disorder diagnoses are less common. ${ }^{1}$ Pediatric sleep disorders include sleeprelated breathing disorders (prevalence: $4-11 \%^{2}$ ), obstructive sleep apnea (OSA; prevalence: $1-4 \%^{2}$ ), restless legs syndrome (RLS, prevalence: $2 \%^{3}$ ), periodic limb movement disorder (PLMD, prevalence: $14 \%^{4}$ ), narcolepsy (prevalence: $\left.0.05 \%^{5}\right)$, insomnia $\left(20-30 \%^{6}\right)$, and parasomnias (prevalence: $14.4 \%^{7}$ ).

Sleep researchers assess pediatric sleep to investigate developmental changes in sleep behavior and neurobiological sleep characteristics. Clinical research aims at identifying discrepancies between clinical populations and typically developing children and adolescents.

received

December 24, 2014 accepted after revision

March 23, 2015

published online

May 11, 2015
Several methods have been developed to cover the needs of clinicians and researchers. The methods differ in terms of information source (objective vs. subjective), time and financial costs, and setting (sleep laboratory vs. habitual environment). Accordingly, they all have their specific field of application.

\section{Questionnaires and Diaries}

In a review from 2011, the authors evaluated currently used questionnaires and scales about sleep in children. ${ }^{8}$ They found 57 instruments in which psychometric testing had been done to some extent. Best ratings for instruments assessing sleep problems in infants ( 1 month-2 years) were obtained by the Sleep and Settle Questionnaire (SSQ), the Maternal Cognitions about Infant Sleep Questionnaire (MCISQ), and the Parental Interactive Bedtime Behavior Scale (PIBBS). These instruments mainly focus on sleep environment and settling. In children (2-11 years) the instruments focus more on sleep-wake patterns, routines, sleep hygiene, and the screening for specific sleep disorders such as (c) 2015 Georg Thieme Verlag KG
Stuttgart . New York

Neurodevelopmental Disorders; Guest Editor, Oskar G. Jenni, MD
DOI http://dx.doi.org/ 10.1055/s-0035-1550232. ISSN 0174-304X. 
insomnia, sleep-related breathing disorders, or periodic limb movement disorder. Toward adolescence (11-18 years) more questions relating to sleepiness or emotional well-being are included. The authors recommend the use of the Bedtime Routines Questionnaire (BRQ), the Tayside Children's Sleep Questionnaire (TCSQ), the Children's Sleep Wake Scale (CSWS), the Behavioral Evaluation of Disorders of Sleep Scale (BEDS), the Pediatric Sleep Questionnaire (PSQ), the Sleeprelated Breathing Disorders Scale (SRBD), the Sleep Disturbance Scale for Children (SDSC), and the Sleep Disorders Inventory for Students-Children (SDIS-C). The latter disposes of a specific version for adolescents (SDIS-A). The Dream Content Questionnaire for Children (ChDCQ) and the Cleveland Adolescent Sleepiness Questionnaire (CASQ) were the only self-reporting instruments with good ratings. A recent preliminary study, showed good psychometric values for a newly developed self-reporting tool for children ${ }^{9}$ : the Children's Report of Sleep Patterns (CRSP). The authors claim that such self-reports might provide complementary information that would not be covered if only relying on parental reports.

Using these instruments, in several clinical populations the prevalence for sleep disorders was found to be increased when compared with the healthy population, that is, in children and adolescents with attention-deficit/hyperactivity disorder (ADHD), in children and adolescents with autistic spectrum disorder (ASD), in children and adolescents with cerebral palsy and in children and adolescents with Down syndrome. ${ }^{10-13}$ The most commonly used instruments to screen for sleep disorders in these children are the Children's Sleep Habit Questionnaire (CSHQ), the SDSC, and the PSQ. The Sleep Self-Report for children and adolescents is mainly used in combination with the CSHQ for parents. ${ }^{14,15}$ The Questionnaire for Children with Severe Psychomotor Impairment (Schlaffragebogen für Kinder mit Neurologischen und Anderen Komplexen Erkrankungen, SNAKE) is a recently developed instrument to assess sleep disorders in children and adolescents with severe psychomotor impairments. ${ }^{16}$ It specifically takes into account impaired perception, intellectual disability, and motor impairment. Another instrument aiming at a specific patient group is the Pediatric Restless Legs Syndrome Severity Scale (P-RLS-SS). ${ }^{17}$ However, the scale has not yet been validated.

While questionnaires and scales ask parents or children to reflect on weekly or monthly sleep behavior, diaries require a daily report of sleep and wake phases. Such diary-based reports were found to be a reliable source of information for sleep start, sleep end, and assumed sleep but not for nocturnal wake time when compared with objective measurements assessed by actigraphy. ${ }^{18}$ In children with sleep disorders this discrepancy between parental report about nocturnal wake time and actigraphy seems to be even more pronounced. $^{19}$

\section{Actigraphy}

Actigraphy uses a watch-like movement sensor to assess habitual sleep-wake patterns. It allows data collection over multiple days and is easily applied in the child's natural environment. At least five nights are required to obtain reliable measures. ${ }^{20}$ The most commonly used devices are the AMI devices (Ambulatory Monitoring Inc. actigraphs: Ardsley, New York, United States), the Mini-Mitter devices (now owned by Phillips-Respironics, Bend, Oregon, United States), and the Cambridge Actiwatch actigraphs (Cambridge, United Kingdom). Across all devices epoch length is most frequently set at 1 minute, less often at 30 seconds. ${ }^{21}$ Sleepwake scoring algorithms, respectively, wake threshold sensitivity typically are device-specific. According to Meltzer et al, ${ }^{21}$ the most commonly used sleep-wake scoring algorithm for the AMI devices is the Sadeh algorithm. ${ }^{22}$ For the MiniMitter and the Cambridge devices the most commonly used wake threshold sensitivity level is the medium sensitivity. The authors suggest that since sleep undergoes major changes in the course of development, devices, and scoring algorithms/ sensitivity levels should be selected age-specifically, based on previously published validation studies. They list 10 validation studies for different age groups which compared actigraphy to "gold standard" sleep measures such as polysomnography (PSG). A more recent validation study used different devices and scoring algorithms in children and adolescents. ${ }^{21}$ Another recent study tested different wake threshold sensitivity levels specifically in 2 to 5 years old children. ${ }^{23}$ Throughout all age groups, devices, epoch lengths, and scoring algorithms, studies consistently reported high sensitivity (proportion of correctly identified sleep epochs) and low specificity (proportion of correctly identified wake epochs). Thus, actigraphy accurately scores sleep periods, but is less suitable for detecting wake periods after sleep onset.

Actigraphy sleep variables such as sleep onset, wake after sleep onset, and sleep offset are determined according to time-related definitions. For example, sleep onset is commonly defined by several consecutive epochs scored as sleep. However, there are no standards for such definitions. To address this concern, Meltzer et $\mathrm{al}^{9}$ provided a list of recommended variable names and definitions that should be considered when reporting results from actigraphy measurements ( - Table $\mathbf{1}$ ). Variables such as bedtime and wake time are assessed using actigraphy markers (button press) or daily sleep logs (-Table 1). Furthermore, sleep logs are needed to determine artifacts such as sleeping in a car or times when the device is removed. Actigraphy has become a widely used method to objectively measure sleep over the past 20 years and has proven to be useful in assessing habitual sleep pattern in children with and without sleep problems. ${ }^{18,24}$ In clinical research, actigraphy is used to investigate sleep and the relationship between sleep and behavioral functions in different clinical populations, for example, children with $\mathrm{ADHD}^{25}$ or children with Down syndrome or Williams syndrome. ${ }^{26}$ In children and adolescents with neurodevelopmental disorders the method allows to detect effects of medication on sleep. ${ }^{27,28}$ However, actigraphy is not a suitable method for the diagnosis of disorders in which sleep is fragmented. For example, the detection of limb movement events in children and adolescents with periodic limb movement disorder is insufficiently accurate. ${ }^{29}$ In children and adolescents with obstructive sleep apnea actigraphy fails to reliably 
Table 1 Recommended variable names and definitions for actigraphy in the pediatric population

\begin{tabular}{|c|c|}
\hline \multicolumn{2}{|l|}{ Reported variables } \\
\hline Bedtime & $\begin{array}{l}\text { Clock time attempted to fall asleep as indicated by either sleep log or event } \\
\text { marker }\end{array}$ \\
\hline Wake time & $\begin{array}{l}\text { Clock time of final awakening in the morning as indicated by either sleep log or } \\
\text { event marker }\end{array}$ \\
\hline Sleep opportunity (time in bed) & Time between bedtime and wake time (reported in min or $\mathrm{h}$ ) \\
\hline \multicolumn{2}{|l|}{ Actigraphy variables } \\
\hline Sleep onset & $\begin{array}{l}\text { Clock time for first of a predetermined number of consecutive min of sleep } \\
\text { following reported bedtime }\end{array}$ \\
\hline Sleep offset & $\begin{array}{l}\text { Clock time for last of a predetermined number of consecutive min of sleep } \\
\text { before reported wake time }\end{array}$ \\
\hline Sleep period & Time between sleep onset and sleep offset (reported in min or h) \\
\hline TST & Duration of sleep in sleep period (reported in min or $\mathrm{h}$ ) \\
\hline Sleep onset latency & Time between bedtime and sleep onset (reported in min) \\
\hline WASO & Number of minutes scored as wake during sleep period \\
\hline Sleep efficiency & Percentage sleep: (TST/time in bed) $\times 100$ \\
\hline Night waking & $\begin{array}{l}\text { Predetermined minimal number of minutes of wake (e.g., }>5 \text { min) preceded } \\
\text { and followed by a predetermined minimal number of minutes of sleep } \\
\text { (e.g., }>15 \text { min) }\end{array}$ \\
\hline Night waking frequency & Number of night wakings \\
\hline Night waking duration & Sum of minutes scored as night waking \\
\hline 24 h sleep duration & Amount of sleep in a 24-h period (reported in min or h) \\
\hline
\end{tabular}

Abbreviations: TST, total sleep time; WASO, wake after sleep onset. Note: Adapted from Meltzer et al, 2012. ${ }^{21}$

identify breathing abnormalities. ${ }^{30}$ For such clinical populations PSG remains the best diagnostic method.

\section{Polysomnogrphaphy}

The American Academy of Sleep Medicine (AASM) manual for the Scoring of Sleep and Associated Events provides technical specifications for PSG recordings and criteria for determining sleep stages, arousals, respiratory events, cardiac events, and movement events. ${ }^{31}$ According to these international guidelines, the electroencephalogram (EEG) should include at least eight electrodes, placed according to the international 10-20 system: bilateral frontal (F4, F3), central $(\mathrm{C} 4, \mathrm{C} 3)$, occipital $(\mathrm{O} 2,01)$, and mastoids (M1, M2). Electrooculogram is recorded using two electrodes (placed $0.5-1 \mathrm{~cm}$ above the right outer canthus and $0.5-1 \mathrm{~cm}$ below the left outer canthus, depending on the children's head size). Electromyogram (EMG) is recorded using submental electrodes. Based on these parameters sleep stages are scored (wakefulness, nonrapid-eye-movement sleep stages 1-3, rapid-eye-movement sleep). The 2007 AASM manual specifies scoring rules for children. Recommended sleep variables are listed in - Table 2 . The scoring rules for sleep arousals are the same for adults and children. The number of arousals and the arousal index are the most currently used variables to quantify sleep disruption (-Table 2). Alternative measures, such as sleep pressure score, cyclic alternating pattern or computerassisted identification of nonvisible arousals may provide complementary information. ${ }^{32}$ For the respiratory monitoring during PSG, the 2007 AASM manual recommends to measure (1) airflow using an oronasal thermal sensor and a nasal air pressure transducer, (2) respiratory effort using esophageal manometry or respiratory inductance plethysmography, (3) oxygen saturation using pulse oximetry, and (4) hypoventilation using transcutaneous or end-tidal $\mathrm{PCO}_{2}$ monitoring. In 2012 the AASM Sleep Apnea Definitions Task Force reviewed evidence for new monitoring technologies and further recommend the use of positive airway pressure (PAP) device flow signal for PAP titration PSG and the use of arterial $\mathrm{PCO}_{2}$ monitoring for hypoventilation. ${ }^{33}$ To detect snoring they recommend several sensors as options: acoustic sensor (e.g., microphone), piezoelectric sensor or nasal pressure transducer. The 2007 AASM manual provides scoring rules for respiratory events such as obstructive apnea, mixed apnea, central apnea, hypopnea, respiratory effortrelated arousals, hypoventilation, and periodic breathing. All scoring rules are specified for children. The 2012 update of the AASM manual ${ }^{33}$ adapted the pediatric scoring rules for central apnea and hypopnea ( - Table 3 ), thereby improving the detection of sleep-disordered breathing in children when compared with previous standards. ${ }^{34,35}$ Recommended respiratory variables are listed in - Table 2.

PSG recordings also include an electrocardiogram. The 2007 AASM manual recommends the use of a two-lead electrocardiograph with electrodes placed on the torso. Scoring rules are the same in adults and in children. Cardiac 
162 Methods in Pediatric Sleep Research and Sleep Medicine Mouthon, Huber

Table 2 Recommended variable names and definitions for polysomnography in the pediatric population

\begin{tabular}{|c|c|}
\hline \multicolumn{2}{|l|}{ Sleep variables } \\
\hline Lights out (A1) & Clock time \\
\hline Lights on (A2) & Clock time \\
\hline TST (A3) & Duration of sleep in sleep period (in min) \\
\hline Total recording time (A4) & Time between lights out and lights on (in min) \\
\hline SL (A5) & Time between lights out and first epoch of sleep (in min) \\
\hline REM sleep latency (A6) & Time between first epoch of sleep and first epoch of REM sleep (in min) \\
\hline WASO (A7) & Wake time during A4-A5 (in min) \\
\hline Sleep efficiency (A8) & Percentage sleep: $(\mathrm{A} 3 / \mathrm{A} 4) \times 100$ \\
\hline Sum of sleep time for each sleep stage (A9) & (in min) \\
\hline Percentage of TST for each sleep stage (A10) & $(\mathrm{A} 9 / \mathrm{A} 3) \times 100$ \\
\hline \multicolumn{2}{|l|}{ Arousal variables } \\
\hline \multicolumn{2}{|l|}{ Number of arousals (B1) } \\
\hline Arl (B2) & $(B 1 \times 60 / A 3)$ \\
\hline \multicolumn{2}{|l|}{ Respiratory variables } \\
\hline \multicolumn{2}{|l|}{ Number of obstructive apneas (C1) } \\
\hline \multicolumn{2}{|l|}{ Number of mixed apneas (C2) } \\
\hline \multicolumn{2}{|l|}{ Number of central apneas (C3) } \\
\hline \multicolumn{2}{|l|}{ Number of hypopneas (C4) } \\
\hline \multicolumn{2}{|l|}{ Number of apneas + hypopneas (C5) } \\
\hline $\mathrm{Al}(\mathrm{C} 6)$ & $(\mathrm{C} 1+\mathrm{C} 2+\mathrm{C} 3) \times 60 / \mathrm{A} 3$ \\
\hline $\mathrm{HI}(\mathrm{C} 7)$ & $\mathrm{C} 4 \times 60 / \mathrm{A} 3$ \\
\hline $\mathrm{AHI}(\mathrm{C} 8)$ & $\mathrm{C} 5 \times 60 / \mathrm{A3}$ \\
\hline Continuous oxygen saturation (C9) & Mean value \\
\hline \multicolumn{2}{|l|}{ Minimum oxygen saturation during sleep (C10) } \\
\hline Occurrence of Cheyne stokes breathing (C11) & Yes/no \\
\hline \multicolumn{2}{|l|}{ Cardiac variables } \\
\hline \multicolumn{2}{|l|}{ Average heart rate during sleep (D1) } \\
\hline \multicolumn{2}{|l|}{ Highest heart rate during sleep (D2) } \\
\hline \multicolumn{2}{|l|}{ Highest heart rate during recording (D3) } \\
\hline Bradycardia (D4) & Yes/no, if present report lowest heart rate observed \\
\hline Asystole (D5) & Yes/no, if present report longest pause observed \\
\hline Sinus tachycardia during sleep (D6) & Yes/no, if present report highest heart rate observed \\
\hline Narrow complex tachycardia (D7) & Yes/no, if present report highest heart rate observed \\
\hline Wide complex tachycardia (D8) & Yes/no, if present report highest heart rate observed \\
\hline Atrial fibrillation (D9) & Yes/no \\
\hline Other arrhythmias (D10) & Yes/no, if present list arrhythmia \\
\hline \multicolumn{2}{|l|}{ Movement variables } \\
\hline \multicolumn{2}{|l|}{ Number of PLMS (E1) } \\
\hline \multicolumn{2}{|l|}{ Number of PLMS with arousals (E2) } \\
\hline PLMSI (E3) & $\mathrm{E} 1 \times 60 / \mathrm{A} 3$ \\
\hline PLMSArI (E4) & $\mathrm{E} 2 \times 60 / \mathrm{A} 3$ \\
\hline
\end{tabular}

Abbreviations: AHI, apnea + hypopnea index; Al, apnea index; Arl, arousal index; HI, hypopnea index; PLMS, periodic limb movements of sleep; PLMSArl, PLMS arousal index; PLMSI, PLMS index; REM, rapid eye movements; SL, sleep latency; TST, total sleep time; WASO, wake after sleep onset. Note: Adapted from Iber et al, 2007. ${ }^{31}$ 
Table 3 Recommended changes to the AASM pediatric respiratory scoring rules

\begin{tabular}{|l|}
\hline Scoring rules for pediatric apnea \\
\hline Score a respiratory event as an apnea if it meets all of the following criteria: \\
\hline There is a drop in the peak signal excursion by $\geq 90 \%$ of the pre-event baseline \\
\hline $\begin{array}{l}\text { The duration of the } \geq 90 \% \text { drop lasts at least the minimum duration as specified by obstructive, mixed, or central apnea } \\
\text { duration criteria }\end{array}$ \\
\hline Scoring rules for pediatric central apnea \\
\hline $\begin{array}{l}\text { Score a respiratory event as central apnea if it meets apnea criteria, is associated with absent inspiratory effort throughout } \\
\text { the entire duration of the event, and at least one of the following criteria is met: }\end{array}$ \\
\hline The event lasts 20 s or longer \\
\hline $\begin{array}{l}\text { The event lasts at least the duration of two breaths during baseline breathing and is associated with an arousal } \\
\text { or } \geq 3 \% \text { oxygen desaturation }\end{array}$ \\
\hline $\begin{array}{l}\text { For infants younger than } 1 \text { y of age, the event lasts at least the duration of two breaths during baseline breathing and is } \\
\text { associated with a decrease in heart rate to less than } 50 \text { beats/min for at least } 5 \text { or less than } 60 \text { beats/min for } \\
\text { at least } 15 s\end{array}$ \\
\hline Scoring rules for pediatric hypopnea \\
\hline Score a respiratory event as a hypopnea if it meets all of the following criteria: \\
\hline The peak signal excursions drop by $\geq 30 \%$ of pre-events baseline \\
\hline The duration of the $\geq 30 \%$ drop lasts for at least 2 breaths \\
\hline There is $\geq 3 \%$ desaturation from pre-event baseline or the event is associated with an arousal \\
\hline
\end{tabular}

Abbreviation: AASM, American Academy of Sleep Medicine.

Note: Adapted from Berry et al, 2012.33

variables are listed in - Table 2. According to the 2007 AASM manual the leg EMG should be recorded using surface electrodes placed longitudinally and symmetrically around the middle of the anterior tibialis muscle so that they are 2 to $3 \mathrm{~cm}$ apart or one-third of the length of the muscle, whichever is shorter. Both legs should be monitored for the presence of leg movements, preferably using separate channels for each leg. Recommended movement variables are listed in - Table 2.

Indications for PSG in the pediatric population are: (1) diagnosis of OSA, (2) clinical evaluation after OSA treatment, (3) diagnosis of PLMD and 4) diagnosis of narcolepsy. ${ }^{36,37}$

According to the American Academy of Pediatrics (AAP) PSG is the current gold standard for the diagnosis of pediatric OSA. ${ }^{38}$ The apnea hypopnea index (AHI) is a commonly used to quantify OSA severity. However, there is no consensus in terms of AHI cutoff values. The current practice is to use an arbitrary cutoff $>3$ standard deviations beyond the mean of the normative $\mathrm{AHI} .{ }^{39}$ Such normative values have been provided for infants, children and adolescents. ${ }^{40,41}$ A recent study investigated whether results obtained with respiratory polygraphy (RP) or PSG are comparable. Although RP would be simpler and more cost-effective, the AHI is underestimated when compared with PSG, notably in children with mild and moderate OSA. ${ }^{42}$ Novel approaches propose the use of algorithms for therapy indication. In addition to parameters derived from PSG such algorithms include factors like the severity of symptoms, risk factors, and the presence of any OSA-related morbidity. ${ }^{43,44}$ Current treatments of pediatric OSA are adenotonsillectomy, positive airway pressure (CPAP or BiPAP), high flow nasal cannula oxygen therapy and administration of anti-inflammatory agents such as montelukast or nasal budesonide, ${ }^{39}$ all significantly reducing the AHI. Treatment effects have been evaluated with follow-up PSG and PAP titration PSG. ${ }^{45-49}$

According to the AASM international classification of sleep disorders, the diagnosis of PLMD requires PSG recordings. One of the diagnostic criteria is a periodic limb movements of sleep index (PLMSI) $>5 / \mathrm{h}^{50}$ Normative data support the clinical periodic limb movement index cutoff of $>5 / h^{51}$ Periodic limb movements during sleep were found to be infrequent in the typically developing children and adolescents. Positive treatment effects of oral or intravenous iron on pediatric PLMD are found in 60 to $70 \%$ of the cases. $^{52,53}$ The diagnosis of RLS in children is challenging, particularly because many young children are unable to describe typical RLS symptoms. Although not essential for diagnosis, a PLMSI $>5 / \mathrm{h}$ is considered supportive evidence. ${ }^{54}$ In children diagnosed with RLS a PLMSI $>5 / \mathrm{h}$ has been found in 63 to $74 \%$ of the cases. ${ }^{55-57}$

As part of the diagnostic evaluation in patients with narcolepsy the Multiple Sleep Latency Test (MSLT) is performed. This test assesses sleep latency and sleep onset rapid eye movement sleep periods (SOREMPs) for four to five daytime naps. A mean sleep latency $<8$ minute and two or more SOREMPs is considered the cutoff for narcolepsy diagnosis. ${ }^{50}$ However, there are no specifications for children. Overnight PSG is systematically performed before MSLT, primarily to rule out other causes of excessive daytime sleepiness. Recent studies in adults and children propose to use night PSG for diagnosis. ${ }^{58,59}$ The authors suggest short REM sleep latency or SOREMP to be diagnostic for narcolepsy. 
In the absence of such findings, however, subsequent MSLT would still be required.

In clinical research, PSG is used to investigate sleep and the relationship between sleep and behavioral functions in different patient populations. For example, children with ADHD were found to have a higher arousal index and a higher PLMSI. ${ }^{60}$ In children with Down syndrome and comorbid OSA cognitive performance was significantly lower than in those without OSA. ${ }^{61}$ Increased sleep onset latencies and reduced REM sleep latencies were found in children and adolescents with depressive disorders ${ }^{62}$ as well as in children with generalized anxiety disorder. ${ }^{63}$

For many research questions comprehensive PSG is not needed. When respiratory and movement parameters are not involved, EEG recordings are sufficient.

\section{Electroencephalography}

In basic and clinical research several sleep EEG measures have been assessed in the course of development. Discrepancies from age norms might be indicative for neurodevelopmental disorders. For example, the relative proportion of non-rapid eye-movement sleep (NREMS) and REMS changes in the course of development. ${ }^{64}$ The percentage of REMS increases from childhood to adolescence. In children and adolescents with ASD the percentage of REMS was found to be significantly lower when compared with typically developing children and adolescents of the same age. ${ }^{65}$

Sleep slow waves during NREMS are a well-established marker for deep sleep. They are generated and maintained by thalamocortical and corticocortical networks. ${ }^{66}$ The activity of these slow waves (slow wave activity, SWA: spectral power $1-4.5 \mathrm{~Hz}$ ) is known to be regulated in a usedependent manner, that is, SWA is increased after prolonged wakefulness in adults ${ }^{67}$ as well as in children and adolescents. ${ }^{68}$ In the course of development the expression of slow waves changes substantially. SWA is known to increase over the first years of life with a peak shortly before puberty and a subsequent decline throughout adolescence. $^{69,70}$

The decay of SWA across the night has been used as a measure for the dissipation of sleep pressure in adults as well as in children and adolescents. ${ }^{67,71,72}$

Another sleep measure is the slope of sleep slow waves which has been proposed to reflect neuronal synchronization in adults, ${ }^{73}$ in children and adolescents, ${ }^{68}$ and in infants. ${ }^{74}$ An overnight decrease in the slope of slow waves was shown to be already present in infants. ${ }^{74}$ In children with continuous spikes and waves during slow wave sleep (CSWS) the absence of this overnight decrease was suggested to reflect nonrestorative sleep ${ }^{75}$ and to be related to neuropsychological deficits in these children. ${ }^{76}$

Sleep spindles are a characteristic feature of NREMS stage 2 and have been described as waxing and waning oscillations between 12 and $15 \mathrm{~Hz}$. Like slow waves they are known to be related to thalamocortical and corticocortical network activity. ${ }^{66}$ In the course of development sleep spindle activity changes in terms of frequency, amplitude, length, and density. ${ }^{41,70}$ In adults as well as in children and adolescents sleep spindles have been related to cognitive abilities. ${ }^{77-80}$

Sleep characteristics cannot only be investigated globally. Interestingly, sleep regulation also shows local, experiencerelated changes. For example, after unilateral sensory stimulation SWA at the corresponding central electrode site over the sensorimotor cortex was found to be higher when compared with the contralateral electrode site. ${ }^{81}$ Frontal slow oscillations (SO: spectral power $<1 \mathrm{~Hz}$ ) were found to be related to declarative memory consolidation. ${ }^{82}$ Recent studies investigating sleep and memory in children could show that frontal SO are correlated with declarative and emotional memory performance in typically developing children, but not in children with ADHD. ${ }^{83,84}$

Another measure using local information from specific electrode sites is EEG coherence. Coherence measures are supposed to reflect brain connectivity. EEG signals are correlated between two recording sites from the same hemisphere (intrahemispheric coherence) or from distinct hemispheres (interhemispheric coherence). ${ }^{85} \mathrm{~A}$ high correlation of neural activity between two recording sites indicates that those regions are directly connected or are both connected to a common third region. Developmental changes in coherence have been assessed from early childhood to adolescence ${ }^{86,87}$ and were suggested to reflect white matter brain maturation. In adolescents changes in intrahemispheric coherence have been related to improved cognitive abilities. ${ }^{88}$ Alterations in coherence were found in children, adolescents, and young adults with ASD. Studies found a reduction in intrahemispheric frontocentral coherence and an increase in intrahemispheric left occipitoparietal and occcipitofrontal coherence. ${ }^{89,90}$ In children and adolescents with major depressive disorder both, intra- and interhemispheric coherence was found to be reduced when compared with typically developing children and adolescents. ${ }^{91}$ In a recent study, the authors calculated coherence values over 19 electrodes (placed according to the 10-20 international system) in infants, children, and adolescents, thereby obtaining topographical coherence maps for different age groups. ${ }^{92}$ They proposed the coherence maps to represent neuronal network maturation.

Mapping EEG measures over the scalp requires a larger number of electrodes than commonly used for sleep EEG recordings. High-density EEG (hdEEG) uses up to 256 electrodes.

\section{High-Density Electroencephalography}

The high number of electrodes opens up entirely new possibilities of EEG signal analysis. Mapping the EEG activity at each electrode creates a topographical picture, visualizing the EEG activity distribution over the scalp. For example, investigating age-related differences in the topographical distribution of SWA revealed an interesting developmental trajectory (-Fig. 1): From early childhood to late adolescence the location of maximal SWA undergoes a shift from posterior toward anterior brain regions. ${ }^{93}$ This pattern corresponds to the course of cortical gray matter maturation. Thus, the SWA 


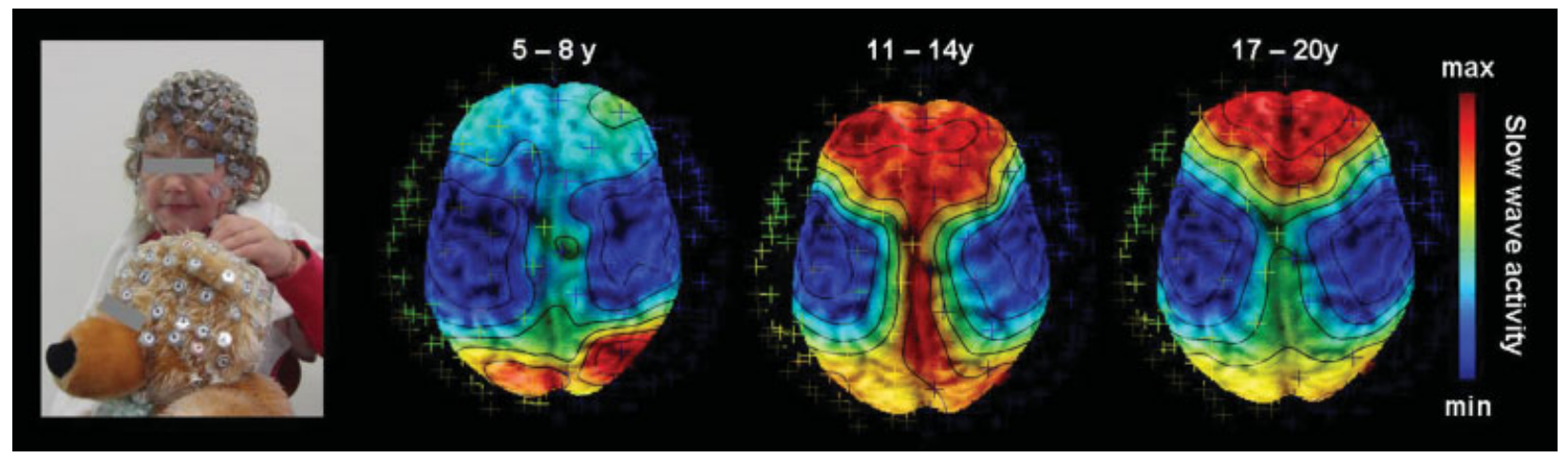

Fig. 1 (Left) A 4-year-old girl wearing a high-density electroencephalography (EEG) net (128 channels; Electrical Geodesics Inc., Eugene, Oregon) and teddy bear wearing a training net. (Right) Topographical maps of relative sleep EEG slow wave activity (1-4.5 Hz) for different age groups superimposed over T1-weighted magnetic resonance images. Crosses surrounding the brain illustrate registered electrode positions on the scalp. Slow wave activity is color coded (maxima in red, minima in blue). Values between electrodes were interpolated.

topography seems to be a marker for the maturational state of the brain. The course of developmental changes in the SWA topography has been related to skill maturation ${ }^{94}$ and showed local gender-specific differences. ${ }^{95}$ This mapping tool might be promising to assess regional differences in brain activity in clinical populations. For example, mapping SWA in children with an ADHD revealed increased SWA over central brain regions when compared with typically developing children and adolescents. ${ }^{96}$ This pattern of SWA distribution in ADHD patients has been hypothesized to reflect altered or delayed brain maturation. Finally, the topographical distribution of EEG activity in other frequency ranges was also investigated. For example, a study investigated the topographical distribution of sleep spindle activity in children and adolescents. ${ }^{97}$ The authors found region-specific positive correlations between spindle activity and cognitive abilities.

hdEEG can also be used to investigate task-related local changes in brain activity. For example, studies have investigated experience-dependent changes in SWA in adults ${ }^{98}$ and more recently in children and adolescents compared with adults. ${ }^{19}$ Interestingly, the task-related local increase of SWA was highest in children, suggesting a critical period of higher neuronal sensitivity to experience when compared with adolescents and adults. An experience-dependent increase in SWA was also shown after 3 weeks of working memory training in children and adolescents. ${ }^{99}$

An even higher spatial resolution of sleep brain activity including deep subcortical structures, for example, the thalamus, can be obtained by simultaneous EEG and functional magnetic resonance imaging (fMRI).

\section{Electroencephalography-Functional Magnetic Resonance Imaging}

EEG-fMRI combines EEG information such as sleep stages or sleep features (e.g., slow waves or sleep spindles) with fMRI network connectivity measures, that is, the coherence of the spontaneous fMRI signal between different brain regions.
This potentially provides new possibilities to investigate sleep brain activity (current methods ${ }^{100}$ ).

So far, only one study used EEG-fMRI to investigate brain network connectivity during sleep in typically developing children. ${ }^{101}$ In children with CSWS-identified networks have been suggested to reflect both spike initiation and propagation pathways. ${ }^{102}$ The deactivations in structures of the default mode network were in line with the concept of epileptiform activity disrupting normal brain function.

The vast majority of studies presented so far involve a correlational approach. To establish causality manipulations are needed. Thus, a promising, not yet established method for future pediatric sleep research is the modulation of sleep by nonpharmacological manipulations.

\section{Modulation of Sleep}

In adults several studies provided evidence for methods successfully enhancing slow waves $\left(\right.$ review $^{103}$ ). The use of transcranial oscillatory direct current stimulation at $0.75 \mathrm{~Hz}$ induced an increase in the slow oscillation EEG activity $(<1 \mathrm{~Hz})$, which was associated with enhanced declarative memory performance, suggesting a causal role for slow waves in memory consolidation. ${ }^{104} \mathrm{~A}$ recent study applying this method in children with ADHD reported similar results. ${ }^{105}$

Another study recently showed that specifically timed acoustic stimuli during slow wave sleep also induce an increase in the slow oscillation EEG activity again associated with enhanced declarative memory performance. ${ }^{106}$ To our knowledge, only one study investigated the feasibility of acoustic stimulation during slow wave sleep in children. In contrast to previous findings in adults, the authors found no effects of acoustic stimulation on EEG activity when applying the same stimulation protocol that had been used for the adult study. ${ }^{107}$ They hypothesize this lack of sensitivity to be due to the higher arousal threshold in children and recommend to consider increased sound levels for future acoustic stimulation studies in children. 
Table 4 Overview of the current methods in sleep medicine and sleep research, limitations, and possible fields of application

\begin{tabular}{|c|c|c|}
\hline Method & Properties & Field of application \\
\hline Questionnaires and diaries & $\begin{array}{l}\text { - Based on parental reports (or self-reports) } \\
\text { - Subjective measures } \\
\text { - Diaries: valid estimates of daily sleep onset, } \\
\text { sleep offset and sleep period, however, } \\
\text { limited accuracy of reported nocturnal wake } \\
\text { times }{ }^{18} \\
\text { - Questionnaires with satisfactory psycho- } \\
\text { metric properties: valid estimates of general } \\
\text { sleep behavior characteristics over extended } \\
\text { time periods (weeks or months) } \\
\text { - Time- and cost-effective data collection and } \\
\text { analysis }\end{array}$ & $\begin{array}{l}\text { - Sleep medicine: identify sleep problems, } \\
\text { screen for sleep disorders } \\
\text { - Sleep research: investigate sleep behavior in } \\
\text { typically developing children and } \\
\text { adolescents }\end{array}$ \\
\hline Actigraphy & $\begin{array}{l}\text { - } \text { Based on movement } \\
\text { - Objective measure } \\
\text { - High sensitivity (detection of sleep) but, low } \\
\text { specificity (detection of wakefulness) } \\
\text { - Data collection over multiple days in the } \\
\text { natural environment } \\
\text { - Simple data analysis using device-specific } \\
\text { software } \\
\text { - Moderate costs }\end{array}$ & $\begin{array}{l}\text { - Sleep medicine: complementary behavioral } \\
\text { information about nocturnal wake times } \\
\text { - Sleep research: investigate sleep-wake pat- } \\
\text { terns in typically developing children and } \\
\text { adolescents }\end{array}$ \\
\hline Polysomnography & $\begin{array}{l}\text { - Based on electrical brain activity, eye move- } \\
\text { ment, submental and leg muscle activity, } \\
\text { respiration and cardiac activity } \\
\text { - Objective measures } \\
\text { - In-laboratory sleep recordings of single nights } \\
\text { - Time intensive and demanding data analysis } \\
\text { - Expensive equipment }\end{array}$ & $\begin{array}{l}\text { - Sleep medicine: diagnosis of periodic limb } \\
\text { movement disorder, obstructive sleep ap- } \\
\text { nea, and narcolepsy (polysomnography and } \\
\text { multiple sleep latency test) } \\
\text { - Sleep research: investigate sleep character- } \\
\text { istics in typically developing children and } \\
\text { adolescents and in clinical populations }\end{array}$ \\
\hline Electroencephalography & $\begin{array}{l}\text { - Based on electrical brain activity, eye move- } \\
\text { ment and submental muscle activity } \\
\text { - Objective measures } \\
\text { - In-laboratory sleep recordings (but also at } \\
\text { home recordings possible, using simplified } \\
\text { equipment) } \\
\text { - Visual sleep scoring } \\
\text { - Expensive equipment }\end{array}$ & $\begin{array}{l}\text { - Sleep research: investigate sleep character- } \\
\text { istics in typically developing children and } \\
\text { adolescents and in clinical populations }\end{array}$ \\
\hline $\begin{array}{l}\text { High-density } \\
\text { electroencephalography }\end{array}$ & $\begin{array}{l}\text { - Based on electrical brain activity, eye move- } \\
\text { - Vent and submental muscle activity } \\
\text { (topualization of brain activity over the scalp } \\
\text { nights } \\
\text { - Objective measures } \\
\text { - Advanced analysis techniques necessary } \\
\text { - In-laboratory sleep recordings of single nights } \\
\text { - Expensive equipment }\end{array}$ & $\begin{array}{l}\text { - Sleep research: investigate sleep character- } \\
\text { istics, with a focus on regional differences, in } \\
\text { typically developing children and adoles- } \\
\text { cents and in clinical populations }\end{array}$ \\
\hline
\end{tabular}

\section{Conclusions and Future Perspectives}

- Table 4 provides an overview of the presented current methods in sleep medicine and sleep research. Limitations and possible fields of application are summarized.

Questionnaires and diaries are a time- and cost-effective method. Subjective parental reports provide information about their children's habitual sleep and sleep problems such as difficulty falling asleep. However, if parents are unable to reliably report or if a more accurate estimation of nocturnal wake times is needed, complementary information provided by actigraphy might be helpful. In children suspected of having PLMD, sleep-related breathing disorders or narcolepsy the gold standard for diagnosis remains PSG.
In pediatric sleep research sleep EEG is a well-established method allowing the analysis of sleep structure (sleep stages) and specific sleep characteristics such as slow waves or spindles. hdEEG additionally allows topographical analysis. fMRI-EEG and the modulation of sleep are not yet established methods. However, especially the modulation of sleep might be a very promising method for future research and clinical application.

\section{References}

1 Owens J. Classification and epidemiology of childhood sleep disorders. Prim Care 2008;35(3):533-546, vii 
2 Lumeng JC, Chervin RD. Epidemiology of pediatric obstructive sleep apnea. Proc Am Thorac Soc 2008;5(2):242-252

3 Picchietti D, Allen RP, Walters AS, Davidson JE, Myers A, FeriniStrambi L. Restless legs syndrome: prevalence and impact in children and adolescents-the Peds REST study. Pediatrics 2007; 120(2):253-266

4 Gingras JL, Gaultney JF, Picchietti DL. Pediatric periodic limb movement disorder: sleep symptom and polysomnographic correlates compared to obstructive sleep apnea. J Clin Sleep Med 2011;7(6):603-9A

5 Peterson PC, Husain AM. Pediatric narcolepsy. Brain Dev 2008; 30(10):609-623

6 Owens JA, Mindell JA. Pediatric insomnia. Pediatr Clin North Am 2011;58(3):555-569

7 Agargun MY, Cilli AS, Sener S, et al. The prevalence of parasomnias in preadolescent school-aged children: a Turkish sample. Sleep 2004;27(4):701-705

8 Spruyt K, Gozal D. Pediatric sleep questionnaires as diagnostic or epidemiological tools: a review of currently available instruments. Sleep Med Rev 2011;15(1):19-32

9 Meltzer LJ, Avis KT, Biggs S, Reynolds AC, Crabtree VM, Bevans KB. The Children's Report of Sleep Patterns (CRSP): a self-report measure of sleep for school-aged children. J Clin Sleep Med 2013;9(3):235-245

10 Hodge D, Carollo TM, Lewin M, Hoffman CD, Sweeney DP. Sleep patterns in children with and without autism spectrum disorders: developmental comparisons. Res Dev Disabil 2014;35(7): 1631-1638

11 Hoffmire CA, Magyar CI, Connolly HV, Fernandez ID, van Wijngaarden E. High prevalence of sleep disorders and associated comorbidities in a community sample of children with Down syndrome. J Clin Sleep Med 2014;10(4):411-419

12 Moreau V, Rouleau N, Morin CM. Sleep, attention, and executive functioning in children with attention-deficit/hyperactivity disorder. Arch Clin Neuropsychol 2013;28(7):692-699

13 Romeo DM, Brogna C, Musto E, et al. Sleep disturbances in preschool age children with cerebral palsy: a questionnaire study. Sleep Med 2014;15(9):1089-1093

14 Owens JA, Maxim R, Nobile C, McGuinn M, Msall M. Parental and self-report of sleep in children with attention-deficit/hyperactivity disorder. Arch Pediatr Adolesc Med 2000;154(6):549-555

15 Sumpter RE, Dorris L, Kelly T, McMillan TM. Pediatric sleep difficulties after moderate-severe traumatic brain injury. J Int Neuropsychol Soc 2013;19(7):829-834

16 Blankenburg M, Tietze AL, Hechler T, et al. Snake: the development and validation of a questionnaire on sleep disturbances in children with severe psychomotor impairment. Sleep Med 2013; 14(4):339-351

17 Arbuckle R, Abetz L, Durmer JS, et al. Development of the Pediatric Restless Legs Syndrome Severity Scale (P-RLS-SS): a patientreported outcome measure of pediatric RLS symptoms and impact. Sleep Med 2010;11(9):897-906

18 Werner H, Molinari L, Guyer C, Jenni OG. Agreement rates between actigraphy, diary, and questionnaire for children's sleep patterns. Arch Pediatr Adolesc Med 2008;162(4):350-358

19 Wilhelm I, Kurth S, Ringli M, et al. Sleep slow-wave activity reveals developmental changes in experience-dependent plasticity. J Neurosci 2014;34(37):12568-12575

20 Acebo C, Sadeh A, Seifer R, et al. Estimating sleep patterns with activity monitoring in children and adolescents: how many nights are necessary for reliable measures? Sleep 1999;22(1): 95-103

21 Meltzer LJ, Montgomery-Downs HE, Insana SP, Walsh CM. Use of actigraphy for assessment in pediatric sleep research. Sleep Med Rev 2012;16(5):463-475

22 Sadeh A, Lavie P, Scher A, Tirosh E, Epstein R. Actigraphic homemonitoring sleep-disturbed and control infants and young chil- dren: a new method for pediatric assessment of sleep-wake patterns. Pediatrics 1991;87(4):494-499

23 Bélanger ME, Bernier A, Paquet J, Simard V, Carrier J. Validating actigraphy as a measure of sleep for preschool children. J Clin Sleep Med 2013;9(7):701-706

24 Werner H, Hunkeler P, Benz C, Molinari L, Huber R, Jenni OG. Valid methods for estimating children's sleep problems in clinical practice. Acta Paediatr 2014;103(12):e555-e557

25 Lee HK, Jeong JH, Kim NY, et al. Sleep and cognitive problems in patients with attention-deficit hyperactivity disorder. Neuropsychiatr Dis Treat 2014;10:1799-1805

26 Ashworth A, Hill CM, Karmiloff-Smith A, Dimitriou D. Cross syndrome comparison of sleep problems in children with Down syndrome and Williams syndrome. Res Dev Disabil 2013;34(5):1572-1580

27 De Crescenzo F, Armando M, Mazzone L, et al. The use of actigraphy in the monitoring of methylphenidate versus placebo in ADHD: a meta-analysis. Atten Defic Hyperact Disord 2014; 6(1):49-58

28 Malow B, Adkins KW, McGrew SG, et al. Melatonin for sleep in children with autism: a controlled trial examining dose, tolerability, and outcomes. J Autism Dev Disord 2012;42(8): 1729-1737, author reply 1738

29 Montgomery-Downs HE, Crabtree VM, Gozal D. Actigraphic recordings in quantification of periodic leg movements during sleep in children. Sleep Med 2005;6(4):325-332

30 O'Driscoll DM, Foster AM, Davey MJ, Nixon GM, Horne RS. Can actigraphy measure sleep fragmentation in children? Arch Dis Child 2010;95(12):1031-1033

31 Iber C, Ancoli-Israel S, Chesson AL, Quan SF. The AASM manual for the scoring of sleep and associated events: rules, terminology and technical specifications. 1st ed. Westchester, IL: American Academy of Sleep Medicine; 2007

32 Paruthi S, Chervin RD. Approaches to the assessment of arousals and sleep disturbance in children. Sleep Med 2010;11(7): 622-627

33 Berry RB, Budhiraja R, Gottlieb DJ, et al; American Academy of Sleep Medicine; Deliberations of the Sleep Apnea Definitions Task Force of the American Academy of Sleep Medicine. Rules for scoring respiratory events in sleep: update of the 2007 AASM Manual for the Scoring of Sleep and Associated Events. J Clin Sleep Med 2012;8(5):597-619

34 Lin $\mathrm{CH}$, Guilleminault C. Current hypopnea scoring criteria underscore pediatric sleep disordered breathing. Sleep Med 2011; 12(7):720-729

35 Nixon GM, Hyde M, Biggs SN, Walter LM, Horne RS, Davey MJ. The impact of recent changes to the respiratory scoring rules in pediatrics. J Clin Sleep Med 2014;10(11):1217-1221

36 Aurora RN, Lamm CI, Zak RS, et al. Practice parameters for the non-respiratory indications for polysomnography and multiple sleep latency testing for children. Sleep 2012;35(11):1467-1473

37 Aurora RN, Zak RS, Karippot A, et al; American Academy of Sleep Medicine. Practice parameters for the respiratory indications for polysomnography in children. Sleep 2011;34(3):379-388

38 Marcus CL, Brooks LJ, Draper KA, et al; American Academy of Pediatrics. Diagnosis and management of childhood obstructive sleep apnea syndrome. Pediatrics 2012;130(3):e714-e755

39 Tan HL, Gozal D, Kheirandish-Gozal L. Obstructive sleep apnea in children: a critical update. Nat Sci Sleep 2013;5:109-123

40 Brockmann PE, Poets A, Poets CF. Reference values for respiratory events in overnight polygraphy from infants aged 1 and 3months. Sleep Med 2013;14(12):1323-1327

41 Scholle S, Zwacka G, Scholle HC. Sleep spindle evolution from infancy to adolescence. Clin Neurophysiol 2007;118(7): 1525-1531

42 Tan HL, Gozal D, Ramirez HM, Bandla HP, Kheirandish-Gozal L. Overnight polysomnography versus respiratory polygraphy in 
the diagnosis of pediatric obstructive sleep apnea. Sleep 2014; 37(2):255-260

43 Gozal D, Kheirandish-Gozal L. New approaches to the diagnosis of sleep-disordered breathing in children. Sleep Med 2010;11(7): 708-713

44 Kaditis A, Kheirandish-Gozal L, Gozal D. Algorithm for the diagnosis and treatment of pediatric OSA: a proposal of two pediatric sleep centers. Sleep Med 2012;13(3):217-227

45 Goldbart AD, Greenberg-Dotan S, Tal A. Montelukast for children with obstructive sleep apnea: a double-blind, placebo-controlled study. Pediatrics 2012;130(3):e575-e580

46 Kheirandish-Gozal L, Gozal D. Intranasal budesonide treatment for children with mild obstructive sleep apnea syndrome. Pediatrics 2008;122(1):e149-e155

47 Marcus CL, Moore RH, Rosen CL, et al; Childhood Adenotonsillectomy Trial (CHAT). A randomized trial of adenotonsillectomy for childhood sleep apnea. N Engl J Med 2013;368(25):2366-2376

48 Marcus CL, Rosen G, Ward SL, et al. Adherence to and effectiveness of positive airway pressure therapy in children with obstructive sleep apnea. Pediatrics 2006;117(3):e442-e451

49 McGinley B, Halbower A, Schwartz AR, Smith PL, Patil SP, Schneider $\mathrm{H}$. Effect of a high-flow open nasal cannula system on obstructive sleep apnea in children. Pediatrics 2009;124(1): 179-188

50 American Academy of Sleep Medicine. The international classification of sleep disorders: diagnostic and coding manual. 2nd ed. Westchester, IL: American Sleep Disorders Association; 2005

51 Marcus CL, Traylor J, Gallagher PR, et al. Prevalence of periodic limb movements during sleep in normal children. Sleep 2014; 37(8):1349-1352

52 Grim K, Lee B, Sung AY, Kotagal S. Treatment of childhood-onset restless legs syndrome and periodic limb movement disorder using intravenous iron sucrose. Sleep Med 2013;14(11):1100-1104

53 Simakajornboon N, Gozal D, Vlasic V, Mack C, Sharon D, McGinley BM. Periodic limb movements in sleep and iron status in children. Sleep 2003;26(6):735-738

54 Picchietti DL, Bruni O, de Weerd A, et al; International Restless Legs Syndrome Study Group (IRLSSG). Pediatric restless legs syndrome diagnostic criteria: an update by the International Restless Legs Syndrome Study Group. Sleep Med 2013;14(12): 1253-1259

55 Kotagal S, Silber MH. Childhood-onset restless legs syndrome. Ann Neurol 2004;56(6):803-807

56 Muhle H, Neumann A, Lohmann-Hedrich K, et al. Childhoodonset restless legs syndrome: clinical and genetic features of 22 families. Mov Disord 2008;23(8):1113-1121, quiz 1203

57 Picchietti DL, Rajendran RR, Wilson MP, Picchietti MA. Pediatric restless legs syndrome and periodic limb movement disorder: parent-child pairs. Sleep Med 2009;10(8):925-931

58 Andlauer $\mathrm{O}$, Moore $\mathrm{H}$, Jouhier $\mathrm{L}$, et al. Nocturnal rapid eye movement sleep latency for identifying patients with narcolepsy/hypocretin deficiency. JAMA Neurol 2013;70(7):891-902

59 Reiter J, Katz E, Scammell TE, Maski K. Usefulness of a Nocturnal SOREMP for Diagnosing Narcolepsy with Cataplexy in a Pediatric Population. Sleep 2014

60 Ferri R, Bruni O, Novelli L, Picchietti MA, Picchietti DL. Time structure of leg movement activity during sleep in attentiondeficit/hyperactivity disorder and effects of levodopa. Sleep Med 2013;14(4):359-366

61 Breslin J, Spanò G, Bootzin R, Anand P, Nadel L, Edgin J. Obstructive sleep apnea syndrome and cognition in Down syndrome. Dev Med Child Neurol 2014;56(7):657-664

62 Lofthouse N, Gilchrist R, Splaingard M. Mood-related sleep problems in children and adolescents. Child Adolesc Psychiatr Clin N Am 2009;18(4):893-916

63 Alfano CA, Reynolds K, Scott N, Dahl RE, Mellman TA. Polysomnographic sleep patterns of non-depressed, non-medicated chil- dren with generalized anxiety disorder. J Affect Disord 2013; 147(1-3):379-384

64 Feinberg I, Davis NM, de Bie E, Grimm KJ, Campbell IG. The maturational trajectories of NREM and REM sleep durations differ across adolescence on both school-night and extended sleep. Am J Physiol Regul Integr Comp Physiol 2012;302(5): R533-R540

65 Buckley AW, Rodriguez AJ, Jennison K, et al. Rapid eye movement sleep percentage in children with autism compared with children with developmental delay and typical development. Arch Pediatr Adolesc Med 2010;164(11):1032-1037

66 Steriade M, Timofeev I. Neuronal plasticity in thalamocortical networks during sleep and waking oscillations. Neuron 2003; 37(4):563-576

67 Achermann P, Borbély AA. Simulation of human sleep: ultradian dynamics of electroencephalographic slow-wave activity. J Biol Rhythms 1990;5(2):141-157

68 Kurth S, Jenni OG, Riedner BA, Tononi G, Carskadon MA, Huber R. Characteristics of sleep slow waves in children and adolescents. Sleep 2010;33(4):475-480

69 Campbell IG, Feinberg I. Longitudinal trajectories of non-rapid eye movement delta and theta EEG as indicators of adolescent brain maturation. Proc Natl Acad Sci U S A 2009;106(13): 5177-5180

70 Jenni OG, Borbély AA, Achermann P. Development of the nocturnal sleep electroencephalogram in human infants. Am J Physiol Regul Integr Comp Physiol 2004;286(3):R528-R538

71 Jenni OG, Carskadon MA. Spectral analysis of the sleep electroencephalogram during adolescence. Sleep 2004;27(4):774-783

72 Tarokh L, Carskadon MA, Achermann P. Dissipation of sleep pressure is stable across adolescence. Neuroscience 2012; 216:167-177

73 Riedner BA, Vyazovskiy VV, Huber R, et al. Sleep homeostasis and cortical synchronization: III. A high-density EEG study of sleep slow waves in humans. Sleep 2007;30(12):1643-1657

74 Fattinger S, Jenni OG, Schmitt B, Achermann P, Huber R. Overnight changes in the slope of sleep slow waves during infancy. Sleep 2014;37(2):245-253

75 Bölsterli BK, Schmitt B, Bast T, et al. Impaired slow wave sleep downscaling in encephalopathy with status epilepticus during sleep (ESES). Clin Neurophysiol 2011;122(9):1779-1787

76 Bölsterli Heinzle BK, Fattinger S, Kurth S, et al. Spike wave location and density disturb sleep slow waves in patients with CSWS (continuous spike waves during sleep). Epilepsia 2014;55(4): 584-591

77 Chatburn A, Coussens S, Lushington K, Kennedy D, Baumert M, Kohler M. Sleep spindle activity and cognitive performance in healthy children. Sleep 2013;36(2):237-243

78 Geiger A, Huber R, Kurth S, Ringli M, Jenni OG, Achermann P. The sleep EEG as a marker of intellectual ability in school age children. Sleep 2011;34(2):181-189

79 Hoedlmoser K, Heib DP, Roell J, et al. Slow sleep spindle activity, declarative memory, and general cognitive abilities in children. Sleep 2014;37(9):1501-1512

80 Schabus M, Hödlmoser K, Gruber G, et al. Sleep spindle-related activity in the human EEG and its relation to general cognitive and learning abilities. Eur J Neurosci 2006;23(7):1738-1746

81 Kattler H, Dijk DJ, Borbély AA. Effect of unilateral somatosensory stimulation prior to sleep on the sleep EEG in humans. J Sleep Res 1994;3(3):159-164

82 Diekelmann S, Born J. The memory function of sleep. Nat Rev Neurosci 2010;11(2):114-126

83 Prehn-Kristensen A, Göder R, Fischer J, et al. Reduced sleepassociated consolidation of declarative memory in attentiondeficit/hyperactivity disorder. Sleep Med 2011;12(7):672-679

84 Prehn-Kristensen A, Munz M, Molzow I, Wilhelm I, Wiesner CD, Baving L. Sleep promotes consolidation of emotional memory in 
healthy children but not in children with attention-deficit hyperactivity disorder. PLoS ONE 2013;8(5):e65098

85 Achermann P, Borbély AA. Coherence analysis of the human sleep electroencephalogram. Neuroscience 1998;85(4):1195-1208

86 Kurth S, Achermann P, Rusterholz T, Lebourgeois MK. Development of Brain EEG Connectivity across Early Childhood: Does Sleep Play a Role? Brain Sci 2013;3(4):1445-1460

87 Tarokh L, Carskadon MA, Achermann P. Developmental changes in brain connectivity assessed using the sleep EEG. Neuroscience 2010;171(2):622-634

88 Tarokh L, Carskadon MA, Achermann P. Early adolescent cognitive gains are marked by increased sleep EEG coherence. PLoS ONE 2014;9(9):e106847

89 Lázár AS, Lázár ZI, Bíró A, et al. Reduced fronto-cortical brain connectivity during NREM sleep in Asperger syndrome: an EEG spectral and phase coherence study. Clin Neurophysiol 2010; 121(11):1844-1854

90 Léveillé C, Barbeau EB, Bolduc C, et al. Enhanced connectivity between visual cortex and other regions of the brain in autism: a REM sleep EEG coherence study. Autism Res 2010;3(5):280-285

91 Armitage R, Hoffmann R, Emslie G, Rintelmann J, Robert J. Sleep microarchitecture in childhood and adolescent depression: temporal coherence. Clin EEG Neurosci 2006;37(1):1-9

92 Chu CJ, Leahy J, Pathmanathan J, Kramer MA, Cash SS. The maturation of cortical sleep rhythms and networks over early development. Clin Neurophysiol 2014;125(7):1360-1370

93 Kurth S, Ringli M, Geiger A, LeBourgeois M, Jenni OG, Huber R. Mapping of cortical activity in the first two decades of life: a highdensity sleep electroencephalogram study. J Neurosci 2010; 30(40):13211-13219

94 Kurth S, Ringli M, Lebourgeois MK, et al. Mapping the electrophysiological marker of sleep depth reveals skill maturation in children and adolescents. Neuroimage 2012;63(2): 959-965

95 Ringli M, Kurth S, Huber R, Jenni OG. The sleep EEG topography in children and adolescents shows sex differences in language areas. Int J Psychophysiol 2013;89(2):241-245
96 Ringli M, Souissi S, Kurth S, Brandeis D, Jenni OG, Huber R. Topography of sleep slow wave activity in children with attention-deficit/hyperactivity disorder. Cortex 2013;49(1):340-347

97 Geiger A, Huber R, Kurth S, Ringli M, Achermann P, Jenni OG. Sleep electroencephalography topography and children's intellectual ability. Neuroreport 2012;23(2):93-97

98 Huber R, Ghilardi MF, Massimini M, Tononi G. Local sleep and learning. Nature 2004;430(6995):78-81

99 Pugin F, Metz AJ, Wolf M, Achermann P, Jenni OG, Huber R. Local increase of sleep SWA after three weeks of working memory training in children and adolescents. Sleep 2015;38(4):607-614

100 Duyn JH. EEG-fMRI Methods for the Study of Brain Networks during Sleep. Front Neurol 2012;3:100

101 Manning JH, Courchesne E, Fox PT. Intrinsic connectivity network mapping in young children during natural sleep. Neuroimage 2013;83:288-293

102 Siniatchkin M, Groening K, Moehring J, et al. Neuronal networks in children with continuous spikes and waves during slow sleep. Brain 2010;133(9):2798-2813

103 Bellesi M, Riedner BA, Garcia-Molina GN, Cirelli C, Tononi G. Enhancement of sleep slow waves: underlying mechanisms and practical consequences. Front Syst Neurosci 2014;8:208

104 Marshall L, Helgadóttir H, Mölle M, Born J. Boosting slow oscillations during sleep potentiates memory. Nature 2006; 444(7119):610-613

105 Prehn-Kristensen A, Munz M, Göder R, et al. Transcranial oscillatory direct current stimulation during sleep improves declarative memory consolidation in children with attention-deficit/ hyperactivity disorder to a level comparable to healthy controls. Brain Stimulat 2014;7(6):793-799

106 Ngo HV, Martinetz T, Born J, Mölle M. Auditory closed-loop stimulation of the sleep slow oscillation enhances memory. Neuron 2013;78(3):545-553

107 Piantoni G, Astill RG, Raymann RJ, Vis JC, Coppens JE, Van Someren EJ. Modulation of $\gamma$ and spindle-range power by slow oscillations in scalp sleep EEG of children. Int J Psychophysiol 2013;89(2):252-258 